\title{
Relationship between Nonalcoholic Fatty Liver Disease and Vitamin D Nutritional Status in Extreme Obesity
}

\author{
Adryana Cordeiro, ${ }^{1}$ Silvia Pereira, ${ }^{1}$ Carlos José Saboya, ${ }^{2}$ and Andrea Ramalho ${ }^{1}$ \\ ${ }^{1}$ Micronutrients Research Center, Federal University of Rio de Janeiro, 21941-590 Rio de Janeiro, RJ, Brazil \\ ${ }^{2}$ Multidisciplinary Center of Bariatric and Metabolic Surgery, 22280-020 Rio de Janeiro, RJ, 22280-020, Brazil \\ Correspondence should be addressed to Adryana Cordeiro; cordeiroadryana@gmail.com
}

Received 15 February 2017; Revised 24 April 2017; Accepted 25 May 2017; Published 8 June 2017

Academic Editor: Kevork M. Peltekian

Copyright (C) 2017 Adryana Cordeiro et al. This is an open access article distributed under the Creative Commons Attribution License, which permits unrestricted use, distribution, and reproduction in any medium, provided the original work is properly cited.

Aim. To evaluate the relationship of nonalcoholic fatty liver disease (NAFLD) with nutritional status of vitamin D in extreme obesity. Methods. Descriptive cross-sectional study in individuals with class III obesity (BMI $\geq 40 \mathrm{~kg} / \mathrm{m}^{2}$ ), aged $\geq 20$ years to $<60$ years. Data were obtained for weight, height, waist circumference (WC), and serum 25-hydroxyvitamin D (25(OH)D) levels. Vitamin D analysis was performed by high performance liquid chromatography (HPLC) and the cutoff points used for its classification were $<$ $20 \mathrm{ng} / \mathrm{mL}$ for deficiency and $20-29.9 \mathrm{ng} / \mathrm{ml}$ for insufficiency. NAFLD gradation was conducted through histological evaluation by liver biopsy. Results. The sample is comprised of 50 individuals ( $86 \%$ female). BMI and average weight were $44.1 \pm 3.8 \mathrm{~kg} / \mathrm{m}^{2}$ and 121.4 $\pm 21.4 \mathrm{~kg}$, respectively. Sample distribution according to serum $25(\mathrm{OH}) \mathrm{D}$ levels showed $42 \%$ of deficiency and $48 \%$ of insufficiency. The diagnosis of NAFLD was confirmed in $100 \%$ of the individuals, of which $70 \%$ had steatosis and $30 \%$ had steatohepatitis. The highest percentage of $25(\mathrm{OH}) \mathrm{D}$ insufficiency was seen in individuals with steatosis $(66 \% / n=21)$ and steatohepatitis $(93 \% / n=16)$. All individuals with steatohepatitis presented VDD $(p<0.01)$. Conclusion. The results of this study showed high prevalence of serum $25(\mathrm{OH}) \mathrm{D}$ inadequacy in individuals with class III obesity, which worsens as the stage of liver disease progresses.

\section{Introduction}

Nonalcoholic fatty liver disease (NAFLD) has become one of the most common chronic liver diseases worldwide [1]. NAFLD is characterized by an accumulation of fat in the liver in the absence of such secondary causes as alcohol abuse, viral hepatitis, and so forth [2], while presenting such wide-ranging histological features as simple macrovesicular steatosis and nonalcoholic steatohepatitis (NASH) that can evolve into fibrosis, cirrhosis, or hepatocellular carcinoma [3].

Vitamin D deficiency (VDD) can result from problems relating to the absorption of vitamin $\mathrm{D}$, hydroxylation due to liver failure, improper dietary intake, or inadequate exposure to sunlight. It is the most prevalent micronutrient deficiency in the world, with a billion people estimated to be deficient [4]. Individuals with obesity, including those suffering from liver disease, are more susceptible to VDD [5]. A potential explanation for this deficiency is, when there is damage of the liver, synthesis of $25(\mathrm{OH}) \mathrm{D}$ may be impaired by the presence of steatosis.

VDD can exacerbate NAFLD at least in part through an inflammatory-mediated pathway, given how vitamin D mediates its intracellular signals via the vitamin $\mathrm{D}$ receptor (VDR), which is constitutively expressed in the liver [6]. VDR expression in the cholangiocytes and hepatocytes of NAFLD sufferers correlated inversely with the extent of the disease [7].

There is limited information on the potential role VDD plays in NAFLD diagnosed via liver biopsy, mainly where NASH is concerned [8]. Thus, the aim of this study is to investigate the relationship between serum 25(OH)D levels and NAFLD staging, as diagnosed via liver biopsy, in extreme obesity. 


\section{Material and Methods}

The study is comprised of 50 individuals with class III obesity (Body mass index $[\mathrm{BMI}] \geq 40 \mathrm{Kg} / \mathrm{m}^{2}$ ), of both sexes, aged $\geq 20$ to $<60$ years, from a clinic specialized in controlling obesity in the municipality of Rio de Janeiro, in the period from January to December 2013. Pregnant women, nursing mothers, patients with malabsorption bowel disorder, acute and chronic infections, and associated endocrinopathies (hypothyroidism and hypocortisolism), subjects with diabetes and on insulin or oral antihyperglycemic agents, individuals who made use of medication or vitamin supplement containing vitamin $\mathrm{D}$, whose alcohol consumption exceeded $20 \mathrm{~g} /$ day in women and $40 \mathrm{~g} /$ day in men, individuals who used drugs that can increase risk factor for NAFLD (amiodarone, corticosteroids, synthetic estrogen, tamoxifen, and nifedipine), and individuals who had alcoholic liver disease, viral hepatitis, autoimmune hepatitis, primary biliary cirrhosis, primary sclerosing cholangitis, liver metabolic disorders, and drug-induced hepatitis or another liver disease that was not NAFLD were excluded from the study.

2.1. Anthropometric Evaluation. Class III obesity classification was based on the World Health Organization (WHO) [9] criteria. BMI calculation was conducted according to anthropometric measurements of weight $(\mathrm{kg})$ and height $\left(\mathrm{m}^{2}\right)[9]$.

Measurement of waist circumference (WC) was performed with the patient standing, relaxed abdomen, arms by the sides, and feet together, and a nonextensible tape measure was used. WC was measured midway between lower rib margin and the superior anterior iliac spine. Measurement was performed at the completion of the individual normal expiration.

2.2. Biochemical Evaluation. For biochemical evaluation, blood sample was obtained by venipuncture after a $12 \mathrm{~h}$ fasting period. Laboratory tests were conducted to evaluate liver function and damage: albumin, aspartate aminotransferase (AST), alanine aminotransferase (ALT), alkaline phosphatase (AP), gamma-glutamyl transpeptidase (GGT), lipid profile [total cholesterol, triglycerides (TG), high-density lipoprotein cholesterol (HDL-c), low density lipoprotein cholesterol (LDL-c)], glucose, and insulin. Determinations of triglycerides, total cholesterol, HDL-c, and glucose were performed by the enzymatic colorimetric method. Reagents for these biochemical evaluations were purchased from Labtest Diagnóstica S.A., Minas Gerais, Brazil. LDL-C fraction was determined in accordance with the Friedewald's formula. Basal insulin was quantified by reversed-phase high performance liquid chromatography (RP-HPLC), (Labtest Diagnóstica S.A., Minas Gerais, Brazil) and the cutoff point used was $24.9 \mathrm{uUI} / \mathrm{mL}$.

Insulin resistance (IR) was identified by HOMA-IR index obtained from the following calculation: HOMA-IR = fasting insulin $(\mathrm{mU} / \mathrm{L}) \times$ fasting blood glucose $(\mathrm{mmol} / \mathrm{L} / 22.5)$. The reference values used were found in the literature for healthy adult individuals and the cutoff point obtained was above 2.5 $[10,11]$.
Vitamin D analysis was conducted in the form of $25(\mathrm{OH}) \mathrm{D}$ and the method used for its quantification was HPLC [12]. Serum values obtained were compared with the cutoff points for normality according to Endocrine Society clinical practice guideline [13]. Thus, levels of serum concentration of $25(\mathrm{OH}) \mathrm{D}$ were classified into deficient $(<20 \mathrm{ng} / \mathrm{ml})$, insufficient $(20-29.9 \mathrm{ng} / \mathrm{ml})$, and sufficient $(\geq 30 \mathrm{ng} / \mathrm{ml}$ and $<100 \mathrm{ng} / \mathrm{ml}$ ). To complete the evaluation of the nutritional status of vitamin $\mathrm{D}$, an investigation was conducted on the sun exposure of the individuals in the study, and the protocol validated by Hanwell and coworkers (2010) [14] was applied.

2.3. Diagnosis of NAFLD: Liver Biopsy. Histological evaluation was conducted through a withdrawal of $4 \mathrm{~mm}$ thickness of the left lobe of the liver via puncture using a $16 \mathrm{G} \times$ $15 \mathrm{~cm}$ Menghini needle (Euromed, Minas Gerais, Brazil). Biopsies were conducted by the medical surgeon along with the bariatric surgery. The overall histological evaluations were performed by the same pathologist, who had no knowledge of the biochemical and clinical data of the patients, through staining of parts by hematoxylin-eosin, Masson's trichrome, and Perls' Prussian blue stain (Interlab, São Paulo, Brazil). Hematoxylin-eosin allows a general view of the acinar architecture, inflammatory infiltrates, and changes in hepatocytes. Masson verifies the presence of fibrosis, whether portal, perisinusoidal, or around centrilobular veins. Perls verifies the presence of iron deposits [15].

Grading of NAFLD and staging of hepatic fibrosis were set in accordance with the proposal of Brunt and coworkers [15]. Grading was performed considering the presence of macrovesicular steatosis (simple steatosis) and necroinflammatory activity (presence of NASH).

\section{Macrovesicular Steatosis}

Grade 0: no steatosis.

Grade 1 (mild): $<33 \%$ of fat accumulation in hepatocytes.

Grade 2 (moderate): between $33 \%$ and $66 \%$ of hepatocytes affected.

Grade 3 (severe): $>66 \%$ of hepatocytes affected.

Necroinflammatory Activity (NASH)

Grade 0: no steatosis.

Grade 1 (mild).

Grade 2 (moderate).

Grade 3 (severe).

Staging of Fibrosis Was Performed in Individuals with NASH

Stage 0: no steatosis.

Stage 1: presence of pericellular or perisinusoidal fibrosis in Zone 3, focal or extensive.

Stage 2: presence of pericellular or perisinusoidal fibrosis in Zone 3 associated with the presence of focal or extensive periportal fibrosis. 
TABLE 1: Mean of age, characteristics anthropometric, and concentration of vitamin D according to gender of the sample $(n=50)$.

\begin{tabular}{lccc}
\hline Characteristics & $\begin{array}{c}\text { Female } \\
(n=43)\end{array}$ & $\begin{array}{c}\text { Male } \\
(n=7)\end{array}$ & $p$ value \\
\hline Age $($ years $)$ & $41.3 \pm 8.6$ & $45.7 \pm 6.6$ & 0.324 \\
BMI $\left(\mathrm{Kg} / \mathrm{m}^{2}\right)$ & $42.7 \pm 3.2$ & $45.8 \pm 2.6$ & 0.765 \\
Weight $(\mathrm{Kg})$ & $118.6 \pm 20.2$ & $124.1 \pm 16.5$ & 0.543 \\
WC $(\mathrm{cm})$ & $121.6 \pm 12.7$ & $119.7 \pm 10.1$ & 0.438 \\
$\begin{array}{l}\text { Vitamin D-25(OH)D } \\
(\mathrm{ng} / \mathrm{mL})\end{array}$ & $22.9 \pm 7.2$ & $17.9 \pm 9.8$ & 0.165 \\
\hline
\end{tabular}

Mean and standard deviation: BMI: body mass index; WC: waist circumference.

Stage 3: presence of pericellular or perisinusoidal fibrosis in Zone 3 and focal or extensive fibrotic bridges.

Stage 4: cirrhosis.

2.4. Statistical Analysis. Statistical calculations were performed by the SPSS program version 17.0. Statistical analyses used were Student's $t$-test or ANOVA to compare continuous variables expressed as mean and standard deviation (SD); Pearson's correlation; and Spearman's correlation between nonparametric variables; association was verified by the Chisquare $\left(\chi^{2}\right)$ test. A 5\% significance level was established.

Odds ratio (OR) and 95\% CI were calculated using logistic regression to determine the risk of NAFLD for nutritional status of 25(OH)D. Model was controlled for BMI and WC.

This study was approved by the Research Ethics Committee of Hospital Universitário Clementino Fraga Filho of Federal University of Rio de Janeiro (Research Protocol no. 011/06-CEP).

\section{Results}

3.1. Description of the Study Population. The sample is comprised of 50 individuals, predominantly for female $(n=$ $43 / 86 \%$ ), without statistical difference between gender. Characteristics anthropometric and concentration of vitamin $\mathrm{D}$ according to gender of the sample were presented in Table 1.

In Table 2, all biochemical variables were shown.

Regarding WC, all patients had values that were above the threshold of $102 \mathrm{~cm}$ (men) and $88 \mathrm{~cm}$ (women), as set by WHO (1998) [16], for high risk of metabolic complications.

3.2. Vitamin D Status in the Subjects of the Study. Prevalence of deficiency and insufficiency of $25(\mathrm{OH}) \mathrm{D}$ in the group studied was $42 \%$ and $48 \%$, respectively, and prevalence of sufficiency was $10 \%$, showing an inadequacy percentage of $90 \%$. According to $25(\mathrm{OH}) \mathrm{D}$ levels stratified by gender, we found a lower mean $(17.9 \pm 9.8 \mathrm{ng} / \mathrm{mL})$ in men as compared to women, found to have a mean of $22.9 \pm 7.2 \mathrm{ng} / \mathrm{mL}(p=$ $0.165)$, without significant difference. Prevalence of vitamin $\mathrm{D}$ deficiency was higher in males $(57.1 \%$ versus $39.6 \% ; p=$ 0.04 ), with virtually no subject meeting the criteria for serum
TABLE 2: Biochemical variables of the sample $(n=50)$.

\begin{tabular}{lc}
\hline Variables & Mean/SD \\
\hline Albumin (g/dL) & $4.2 \pm 0.5$ \\
AST (U/L) & $25.1 \pm 18.3$ \\
ALT (U/L) & $27.4 \pm 15.9$ \\
AP (U/L) & $76.1 \pm 23.4$ \\
GGT (U/L) & $33.4 \pm 21.8$ \\
TG (mg/dL) & $132.0 \pm 50.9$ \\
Cholesterol (mg/dL) & $197.7 \pm 36.1$ \\
LDL-c (mg/dL) & $121.8 \pm 34.5$ \\
HDL-c (mg/dL) & $48.3 \pm 11.86$ \\
Insulin (mcU/mL) & $19.0 \pm 12.5$ \\
Glucose (mg/dL) & $100.2 \pm 19.0$ \\
HOMA-IR & $4.5 \pm 4.7$ \\
\hline
\end{tabular}

Mean and standard deviation: ALT: alanine aminotransferase; AP: alkaline phosphatase; AST: aspartate aminotransferase; GGT: gamma-glutamyl transpeptidase; LDL-C: low density lipoprotein cholesterol; HDL-C: highdensity lipoprotein cholesterol; HOMA-IR: homeostatic model assessment insulin resistance; TG: triglycerides.

sufficiency of vitamin D according to the cutoff point for normality proposed by Holick and coworkers (2011) [13].

Assessing the time of sun exposure, a mean was $13.2 \pm 5.2$ minutes using the validated protocol.

3.3. Relation between Vitamin D Status and Biochemical Indicators of Liver Function and Damage. Means of AP, ALT, AST, GGT, and albumin did not present significant statistical difference between the groups of nutritional status of vitamin $\mathrm{D}$, as shown in Table 3.

3.4. Diagnosis of NAFLD and the Relationship with Serum Concentrations of Biochemical Indicators of Liver Function and Damage. Diagnosis of NAFLD was confirmed in $100 \%$ of the patients according to histological evaluation by liver biopsy: $70 \%$ of the individuals had steatosis and 30\% had steatohepatitis. Among the individuals who had steatohepatitis, $13 \%$ presented with fibrosis.

Table 4 shows the mean serum concentrations of biochemical indicators of liver function and damage according to staging of NAFLD. GGT activity was higher in individuals with steatohepatitis (significantly for steatohepatitis with fibrosis versus steatosis); ALT and AST liver enzyme activities were within the normality standard as well as AP levels. Albumin levels were significantly lower in individuals with steatohepatitis with fibrosis.

3.5. Diagnosis of NAFLD and the Relationship with Anthropometric Variables and HOMA-IR. Individuals with steatohepatitis with fibrosis had weight, WC, and BMI levels without significant difference when compared with steatosis alone (Table 5). Besides, in these subjects with presence of fibrosis, we noted that HOMA-IR value was higher $(5.7 \pm 1.2)$ than individuals with steatosis $(4.6 \pm 0.9)[p=0.031]$.

3.6. Correlation between Staging of NAFLD with Serum Concentrations of $25(\mathrm{OH}) \mathrm{D}$. When correlating the degree of 
TABLE 3: Mean serum concentrations of biochemical indicators of liver tests in relation to the nutritional status of vitamin D.

\begin{tabular}{|c|c|c|c|c|}
\hline \multirow[b]{2}{*}{ Variables } & \multicolumn{4}{|c|}{ Nutritional status of vitamin D } \\
\hline & $\begin{array}{c}\text { Deficiency } \\
n=20\end{array}$ & $\begin{array}{c}\text { Insufficiency } \\
n=24\end{array}$ & $\begin{array}{l}\text { Sufficiency } \\
\quad n=6\end{array}$ & $p$ value \\
\hline $\operatorname{AST}(\mathrm{U} / \mathrm{L})$ & $28.1 \pm 22.8$ & $23.5 \pm 14.9$ & $19.4 \pm 5.3$ & 0.672 \\
\hline $\operatorname{ALT}(\mathrm{U} / \mathrm{L})$ & $29.1 \pm 17.5$ & $26.9 \pm 15.8$ & $22.4 \pm 9.9$ & 0.587 \\
\hline $\mathrm{AP}(\mathrm{U} / \mathrm{L})$ & $72.7 \pm 18.2$ & $81.2 \pm 26.1$ & $65.6 \pm 28.1$ & 0.234 \\
\hline GGT (U/L) & $39.2 \pm 25.7$ & $28.9 \pm 17.1$ & $30.3 \pm 22.8$ & 0.212 \\
\hline Albumin (U/L) & $4.4 \pm 0.6$ & $4.1 \pm 0.3$ & $4.1 \pm 0.3$ & 0.287 \\
\hline
\end{tabular}

ANOVA: mean and standard deviation. AST: aspartate aminotransferase; ALT: alanine aminotransferase; AP: alkaline phosphatase; GGT: gamma-glutamyl transpeptidase.

TABLE 4: Mean deviation serum concentrations of biochemical indicators of liver tests according to the staging of NAFLD.

\begin{tabular}{|c|c|c|c|c|}
\hline \multirow[b]{2}{*}{ Variables } & \multicolumn{4}{|c|}{ Staging of NAFLD by liver biopsy } \\
\hline & $\begin{array}{l}\text { Steatosis } \\
(n=31)\end{array}$ & $\begin{array}{l}\text { Steatohepatitis without fibrosis } \\
\qquad(n=17)\end{array}$ & $\begin{array}{l}\text { Steatohepatitis with fibrosis } \\
\qquad(n=2)\end{array}$ & $p$ value \\
\hline AST (U/L) & $22.9 \pm 12.8$ & $29.9 \pm 26.7$ & $30.2 \pm 7.6$ & 0.410 \\
\hline $\operatorname{ALT}(\mathrm{U} / \mathrm{L})$ & $27.1 \pm 15.5$ & $28.2 \pm 17.5$ & $28.9 \pm 10.3$ & 0.343 \\
\hline $\mathrm{AP}(\mathrm{U} / \mathrm{L})$ & $75.1 \pm 24.9$ & $78.3 \pm 20.2$ & $73.1 \pm 1.4$ & 0.722 \\
\hline GGT (U/L) & $24.9 \pm 17.8$ & $42.6 \pm 27.7$ & $62.4 \pm 14.6$ & 0.044 \\
\hline Albumin $(\mathrm{U} / \mathrm{L})$ & $4.5 \pm 0.7$ & $3.9 \pm 0.3$ & $3.3 \pm 0.2$ & 0.029 \\
\hline
\end{tabular}

ANOVA: mean and standard deviation. Alb: albumin; ALT: alanine aminotransferase; AP: alkaline phosphatase; AST: aspartate aminotransferase; GGT: gamma-glutamyl transpeptidase.

TABLE 5: Mean/deviation of anthropometric variables according to the staging of NAFLD.

\begin{tabular}{lccc}
\hline \multirow{2}{*}{ Variables } & \multicolumn{3}{c}{ Staging of NAFLD by liver biopsy } \\
& Steatosis alone & $\begin{array}{c}\text { Steatohepatitis } \\
\text { with fibrosis }\end{array}$ & $p$ value \\
\hline BMI $\left(\mathrm{Kg}^{2}\right)$ & $43.7 \pm 1.9$ & $45.6 \pm 2.2$ & 0.447 \\
Weight $(\mathrm{Kg})$ & $145.9 \pm 36.5$ & $148.5 \pm 47.4$ & 0.209 \\
WC $(\mathrm{cm})$ & $132.3 \pm 11.5$ & $144.0 \pm 18.3$ & 0.087
\end{tabular}

Student's $T$-test: mean and standard deviation: BMI: body mass index; WC: waist circumference.

staging of NAFLD with serum concentrations of 25(OH)D, the current study demonstrated that, in individuals with steatosis, steatohepatitis without fibrosis and steatohepatitis with fibrosis the percentages of sufficiency, insufficiency, and deficiency were $19 \%, 66 \%$, and $15 \%$; $0 \%, 18 \%$, and $82 \%$; $0 \%, 0 \%$, and $100 \%$, respectively. Data showed the highest percentage of deficiency in the group with steatohepatitis with fibrosis.

A significant difference was observed in the serum concentrations of $25(\mathrm{OH}) \mathrm{D}$ when the staging of NAFLD was determined by liver biopsy $(p<0.01)$. The highest mean was found in a milder degree of NAFLD (steatosis) but it showed inadequacy, and the lowest mean of $25(\mathrm{OH}) \mathrm{D}$ was found in steatohepatitis with fibrosis, as shown in Figure 1.

3.7. Association between Vitamin D and NAFLD by Logistic Regression Analysis. An association between vitamin D and NAFLD according to binary logistic regression analyses was observed. After adjustment for BMI and WC, the OR for

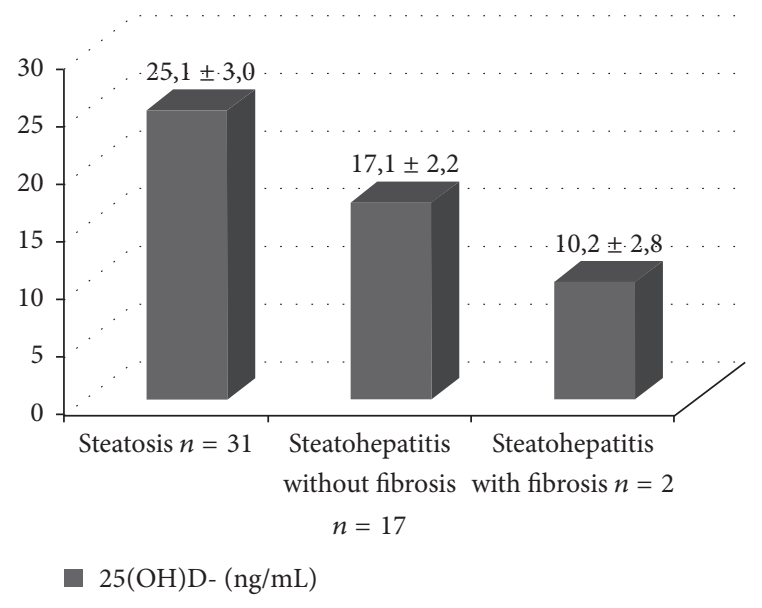

FIgURE 1: Mean serum concentrations of vitamin D [25(OH)D] by staging of NAFLD by liver biopsy. ANOVA $(p<0,01)$.

NAFLD in the deficiency compared with sufficiency of serum concentrations of vitamin D was $1.92(95 \%$ CI $1.38,1.98)[p=$ 0.001 ] and the insufficiency compared with sufficiency was $1.53(95 \%$ CI $1.27,1.78)[p=0.002]$.

3.8. Correlation between Serum Concentrations of Vitamin D and Anthropometric/Biochemical Parameters. When serum concentrations of vitamin D were considered, a significant negative correlation was found with TG, HOMA-IR, insulin, and WC $(r=-0.384, p<0.01 ; r=-0.330, p<0.05$; $r=-0.332, p=0.01 ; r=-0.320, p=0.02$, resp.). 


\section{Discussion}

The results of this study show that the lowest serum vitamin D concentrations in the form of $25(\mathrm{OH}) \mathrm{D}$ were found in steatohepatitis diagnosed by liver biopsy, a gold standard method of diagnosis. We demonstrated that VDD is prevalent in patients with NAFLD, mainly in more histologically severe stages of the disease, thus suggesting that vitamin D may play a role in the development and progression of liver disease, partly via inhibition of vitamin D's anti-inflammatory properties [17]. Furthermore, vitamin D's immunomodulatory properties may help explain the impact this vitamin has on the progression and severity of NAFLD [18]. As the liver is a key organ in vitamin $\mathrm{D}$ synthesis, by transforming vitamin $\mathrm{D}_{3}$ into $25(\mathrm{OH}) \mathrm{D}$, impairment of the organ may explain why vitamin $\mathrm{D}$ is found to be inadequate in most liver diseases. A deficit in vitamin D-VDR axis signaling may represent an aggravating factor for these diseases, because VDR expression is low or absent from hepatocytes and the association between vitamin D-VDR axis and pathophysiology may be the result of an alteration in signals from nonparenchymal liver cells or hepatic cells, especially where NAFLD is concerned [19]. Given how low serum 25(OH)D levels are associated with a progression in hepatic fibrosis, the risk of these individuals developing hepatocellular carcinoma increases, since vitamin $\mathrm{D}$ acts as an inhibitor of hepatic fibrosis [20] and the expression of detoxifying enzyme in the liver. Additionally, VDR is expressed in hepatic stellate cells, which are major producers of cellular matrix and the main indicators of liver fibrosis [21].

We found that VDD had an independent relationship (OR 1.92; 95\% CI 1.38, 1.98) with NAFLD, suggesting that serum 25(OH)D levels protected against NAFLD after adjusting for WC and BMI. VDD could potentiate the metabolic pathways of NAFLD pathogenesis, including immunological, hormonal, and cellular differentiation mechanisms, affecting adipocytokines and proinflammatory cytokines, which are secreted by adipose tissue (AT) and are significant in the progression of NAFLD $[22,23]$. Recent studies suggest that VDD is a significant risk factor for the development of NAFLD [24] and indicate that vitamin D's anti-inflammatory and immune modulatory properties may provide plausible mechanisms by which vitamin D may impact NAFLD progression and severity. Moreover, a study from China found that VDD can positively regulate endogenous fatty acid synthesis in NASH via impaired enterohepatic circulation and the administration of $1,25(\mathrm{OH})_{2} \mathrm{D}_{3}$ corrected NASH phenotypes in accordance with inflammation and hepatic lipogenesis [25]. In animal studies vitamin D was found to play an important role in the regulation of oxidative stress, the production of proinflammatory cytokines [26], hepatocyte apoptosis, and hepatic fibrosis [27].

In our study, the individuals with higher BMI and body weight were those who suffered steatohepatitis with fibrosis, and further studies highlight how there is a strong association between excessive adiposity and NAFLD and IR. This association causes predisposition to systemic hypertension, dyslipidemia, and inflammation. Additionally, the association between these clinical findings and excess AT involves metabolic and inflammatory mechanisms [28]. Although VDD mechanisms that contribute to the deposition of fat in the liver are not yet well understood, studies show that VDD may be involved in the regulation of insulin action and could relate to a decrease in secretion of this hormone [29], malfunction in the level of insulin reception [30], and the induction of subclinical inflammation [31]. As the association between IR and NAFLD is well-established in the literature, it is reasonable to assume that there is some connection between VDD and liver disease, as reported in the experimental study developed by Roth et al. (2012) [26] in which VDD caused IR due to an increase in the gene expression of liver resistin, inflammatory liver regulation, and genes of oxidative stress. Vitamin D also modulates the metabolism of free fatty acids (FFAs) acting on peroxisome proliferator-activated receptor (PPAR- $\gamma$ ), thereby relieving FFA-induced insulin resistance in vitro. Consequently the increased FFAs flowing in the bloodstream could promote fat deposition into the hepatocytes and the progression of NAFLD on condition of VDD [32].

Several studies have shown the relationship between obesity and inadequacy of VD [33-35]; both interact synergistically to influence the risk of insulin resistance [36]. Low serum of $25(\mathrm{OH}) \mathrm{D}$ concentrations is found to be inversely correlated with measures of obesity, including BMI $\left(\geq 30 \mathrm{~kg} / \mathrm{m}^{2}\right)$, fat mass, and WC [37, 38]. A bidirectional genetic study developed suggested that high BMI led to lower $25(\mathrm{OH}) \mathrm{D}$; each unit increase in $\mathrm{BMI}$ is being associated with $1.15 \%$ lower concentration of $25(\mathrm{OH}) \mathrm{D}$ [39]. In our study we found a negative correlation between WC and serum vitamin $\mathrm{D}$ levels, reinforcing findings which demonstrate that more important than the total amount of body fat is its distribution, and the presence of fat in the abdominal region is a predictive factor for the worsening of VDD [40]. In stage of obesity, AT undergoes hypertrophic enlargement which results in an unbalanced blood flow leading to inflammation and macrophage infiltration [41], and as a consequence there is a reduction in adiponectin secretion and an increase in proinflammatory cytokines [42]. Obesity is commonly linked to an upregulation of proinflammatory molecules and downregulation of anti-inflammatory molecules [43]; individuals with both high SAT and high VAT have an approximately threefold prevalence of VDD compared with those with both low SAT and low VAT [44]. 1,25(OH) $)_{2} \mathrm{D}_{3}$ inhibits chronic inflammation in AT by regulating approximately 140 genes favoring oxidative stress and inflammatory biomarkers; reducing IL- 6 , IL-8, and IL- $1 \beta$; and inhibiting of NF- $\kappa \beta$ [20, 45-47].

Our study is comprised of a majority of women, which matched the research of Benoit et al. (2014) [48], where $73-80 \%$ of the individuals attending a clinic specialized in controlling obesity were females; therefore gender was not confounding factor. A higher prevalence of VDD was found in men, without adequate vitamin D nutritional status, despite the fact that these individuals resided in a city with a great deal of sunlight. Our findings confirm those of Cabral et al. (2013) [49], who reported a high prevalence of vitamin D deficiency in men, similar to those in our study. Some studies report a high prevalence of VDD worldwide [50-52], even 
in countries at low latitudes, where there is usually higher UVB irradiation, resulting in conditions suitable for synthesis of the vitamin. A recent study involving Brazilian women of reproductive age presented a mean of $25(\mathrm{OH}) \mathrm{D}$ as 23.8 $\pm 8.7 \mathrm{ng} / \mathrm{mL}$ [53], values very close to ours. A recent study carried out in the United Kingdom evaluating individuals with class III obesity in preoperative bariatric surgery [54] showed a $90 \%$ percentage of serum vitamin D deficiency, assessed in the form of $25(\mathrm{OH}) \mathrm{D}$ in the sample studied, data similar to our findings. Among the indicators of hepatocellular damage most widely used in the literature are GGT and AP transaminases [55], but in this study mean levels of ALT, AST, and AP among individuals with hepatic steatosis and steatohepatitis showed no significant differences. Although many studies have reported an increase in indicators of liver function and damage in individuals with various degrees of NAFLD [56,57], these enzymes generally are normal in over $78 \%$ of individuals [58]. Regarding GGT values, our study showed values statistically higher in individuals with steatohepatitis, especially in those with fibrosis, which suggests that the worsening of liver disease may be a possible explanation for increased GGT as liver fibrosis progresses [59].

Strengths and Weaknesses of the Study. The present study has some limitations: (1) the number of patients evaluated in the research; (2) the noninclusion of a control group. However, there is still a dearth of literature regarding studies that jointly evaluate the relationship between VDD and NAFLD according to severity of disease. Additionally, our findings highlight the relevance of the NAFLD classification method and staging of hepatic fibrosis through liver biopsy, considering the gold standard for its high diagnostic accuracy.

\section{Conclusion}

The results of this study show high prevalence of serum $25(\mathrm{OH}) \mathrm{D}$ inadequacy in individuals with class III obesity, which worsens as the stage of liver disease progresses.

Monitoring vitamin D nutritional status in sufferers of class III obesity and NAFLD is important for the management of their health. Further investigations are needed in order to provide more information regarding new mechanisms in NAFLD physiopathology when associated with VDD.

\section{Conflicts of Interest}

The authors declare that they have no conflicts of interest.

\section{Authors' Contributions}

Adryana Cordeiro participated in coordination of the study, performed the statistical analysis, drafted the manuscript, and has primary responsibility for the final content. Adryana Cordeiro, Silvia Pereira, Carlos José Saboya, and Andrea Ramalho contributed to study conception and design. Silvia Pereira and Carlos José Saboya contributed to acquisition of data analysis. Adryana Cordeiro and Andrea Ramalho contributed to data analysis. Andrea Ramalho helped drafting the manuscript besides coordinating it and giving the final approval of the version to be published. All authors read and approved the final manuscript.

\section{Acknowledgments}

The authors acknowledge the financing of Fundação de Amparo à Pesquisa do Estado do Rio de Janeiro (FAPERJ) and acknowledge the contribution from Multidisciplinary Center of Bariatric and Metabolic Surgery Team that kindly provided the patients to be investigated. Finally, the cooperation of the subjects who took part in the study was greatly appreciated.

\section{References}

[1] N. Chalasani, Z. Younossi, J. E. Lavine et al., "The diagnosis and management of non-alcoholic fatty liver disease: practice guideline by the American Gastroenterological Association, American Association for the Study of Liver Diseases, and American College of Gastroenterology," Gastroenterology, vol. 142, no. 7, pp. 1592-1609, 2012.

[2] M. J. Armstrong, L. A. Adams, A. Canbay, and W.-K. Syn, "Extrahepatic complications of nonalcoholic fatty liver disease," Hepatology, vol. 59, no. 3, pp. 1174-1197, 2014.

[3] G. Vernon, A. Baranova, and Z. M. Younossi, "Systematic review: the epidemiology and natural history of non-alcoholic fatty liver disease and non-alcoholic steatohepatitis in adults," Alimentary Pharmacology and Therapeutics, vol. 34, no. 3, pp. 274-285, 2011.

[4] M. Wacker and M. F. Holiack, "Vitamin D-effects on skeletal and extraskeletal health and the need for supplementation," Nutrients, vol. 5, no. 1, pp. 111-148, 2013.

[5] A. Obispo Entrenas, D. Legupin Tubio, F. Lucena Navarro et al., "Relationship between vitamin D deficiency and the components of metabolic syndrome in patients with morbid obesity, before and 1 year after laparoscopic Roux-en-Y gastric bypass or sleeve gastrectomy," Obesity Surgery, vol. 27, no. 5, pp. 1222-1228, 2017.

[6] S. Han, T. Li, E. Ellis, S. Strom, and J. Y. L. Chiang, "A novel bile acid-activated vitamin $\mathrm{D}$ receptor signaling in human hepatocytes," Molecular Endocrinology, vol. 24, no. 6, pp. 11511164, 2010.

[7] I. Barchetta, S. Carotti, G. Labbadia et al., "Liver vitamin D receptor, CYP2R1, and CYP27A1 expression: relationship with liver histology and vitamin D3 levels in patients with nonalcoholic steatohepatitis or hepatitis C virus," Hepatology, vol. 56, no. 6, pp. 2180-2187, 2012.

[8] R. M. Kwok, D. M. Torres, and S. A. Harrison, "Vitamin D and nonalcoholic fatty liver disease (NAFLD): is it more than just an association?" Hepatology, vol. 58, no. 3, pp. 1166-1174, 2013.

[9] World Health Organization (WHO), World Health Statistics 2011, World Health Organization (WHO), 2014, http://www .who.int/healthinfo/statistics/en/index.html.

[10] C. M. Durward, T. J. Hartman, and S. M. Nickols-Richardson, "All-cause mortality risk of metabolically healthy obese individuals in NHANES III," Journal of Obesity, vol. 2012, Article ID 460321, 12 pages, 2012.

[11] G. Calori, G. Lattuada, L. Piemonti et al., "Prevalence, metabolic features, and prognosis of metabolically healthy obese Italian individuals: the cremona study," Diabetes Care, vol. 34, no. 1, pp. 210-215, 2011. 
[12] J. G. Dorsey, "Introduction to modern liquid chromatography," Journal of the American Chemical Society, vol. 132, no. 26, p. 9220, 2010.

[13] M. F. Holick, N. C. Binkley, H. A. Bischoff-Ferrari et al., "Evaluation, treatment, and prevention of vitamin D deficiency: an endocrine society clinical practice guideline," The Journal of Clinical Endocrinology \& Metabolism, vol. 96, no. 7, pp. 19111930, 2011.

[14] H. E. C. Hanwell, R. Vieth, D. E. C. Cole et al., "Sun exposure questionnaire predicts circulating 25-hydroxyvitamin D concentrations in Caucasian hospital workers in southern Italy," Journal of Steroid Biochemistry and Molecular Biology, vol. 121, no. 1-2, pp. 334-337, 2010.

[15] E. M. Brunt, C. G. Janney, A. M. Di Bisceglie, B. A. Neuschwander-Tetri, and B. R. Bacon, "Nonalcoholic steatohepatitis: a proposal for grading and staging the histological lesions," American Journal of Gastroenterology, vol. 94, no. 9, pp. 2467-2474, 1999.

[16] World Health Organization (WHO), "Obesity: preventing and managing the global epidemic," Tech. Rep., WHO Consultation on Obesity, Geneva, Switzerland, 1998.

[17] X. Wang, W. Li, Y. Zhang, Y. Yang, and G. Qin, "Association between vitamin $\mathrm{D}$ and non-alcoholic fatty liver disease/nonalcoholic steatohepatitis: results from a meta-analysis," International Journal of Clinical and Experimental Medicine, vol. 8, no. 10, pp. 17221-17234, 2015.

[18] M. T. Kitson and S. K. Roberts, "D-livering the message: the importance of vitamin D status in chronic liver disease," Journal of Hepatology, vol. 57, no. 4, pp. 897-909, 2012.

[19] S. Zúñiga, D. Firrincieli, C. Housset, and N. Chignard, "Vitamin $\mathrm{D}$ and the vitamin D receptor in liver pathophysiology," Clinics and Research in Hepatology and Gastroenterology, vol. 35, no. 4, pp. 295-302, 2011.

[20] N. Ding, R. T. Yu, N. Subramaniam et al., "A vitamin D receptor/SMAD genomic circuit gates hepatic fibrotic response," Cell, vol. 153, no. 3, pp. 601-613, 2013.

[21] V. Fedirko, T. D. Salles, and C. Bamia, "Prediagnostic circulating vitamin D levels and risk of hepatocellular carcinoma in European populations: a nested case-control study," Hepatology, vol. 60, no. 4, pp. 1222-1230, 2014.

[22] N. Sharifi, R. Amani, E. Hajiani, and B. Cheraghian, "Does vitamin D improve liver enzymes, oxidative stress, and inflammatory biomarkers in adults with non-alcoholic fatty liver disease? a randomized clinical trial," Endocrine, vol. 47, no. 1, pp. 70-80, 2014.

[23] N. Wang, Q. Li, B. Han, and Y. Lu, "Follicle-stimulating hormone is associated with non-alcoholic fatty liver disease in Chinese women over 55 years old," Journal of Gastroenterology and Hepatology, vol. 31, no. 6, 2015.

[24] A. A. Mohamed, S. Mahmoud, R. Ahmed et al., "Vitamin D is it a key in Egyptian NAFLD pathogenesis?" Journal of Gastroenterology and Hepatology Research, vol. 4, no. 5, pp. 1605-1609, 2015.

[25] M. Kong, L. Zhu, L. Bai et al., "Vitamin D deficiency promotes nonalcoholic steatohepatitis through impaired enterohepatic circulation in animal model," American Journal of Physiology Gastrointestinal and Liver Physiology, vol. 307, no. 9, pp. G883G893, 2014.

[26] C. L. Roth, C. T. Elfers, D. P. Figlewicz et al., "Vitamin D deficiency in obese rats exacerbates nonalcoholic fatty liver disease and increases hepatic resistin and toll-like receptor activation," Hepatology, vol. 55, no. 4, pp. 1103-1111, 2012.
[27] J. J. Potter, X. Liu, A. Koteish, and E. Mezey, "1,25-dihydroxyvitamin D3 and its nuclear receptor repress human $\alpha_{1}(\mathrm{I})$ collagen expression and type I collagen formation," Liver International, vol. 33, no. 5, pp. 677-686, 2013.

[28] F. Item and D. Konrad, "Visceral fat and metabolic inflammation: the portal theory revisited," Obesity Reviews, vol. 3, no. supplement 2, pp. 30-39, 2012.

[29] J. A. Alvarez and A. Ashraf, "Role of vitamin D in insulin secretion and insulin sensitivity for glucose homeostasis," International Journal of Endocrinology, vol. 2010, Article ID 351385, 18 pages, 2010.

[30] C. Calle, B. Maestro, and M. García-Arencibia, "Genomic actions of 1,25-dihydroxyvitamin $\mathrm{D} 3$ on insulin receptor gene expression, insulin receptor number and insulin activity in the kidney, liver and adipose tissue of streptozotocin-induced diabetic rats," BMC Molecular Biology, vol. 18, no. 9, article 65, 2008.

[31] A. G. Pittas, M. Chung, T. Trikalinos et al., "Systematic review: vitamin D and cardiometabolic outcomes," Annals of Internal Medicine, vol. 152, no. 5, pp. 307-314, 2010.

[32] I. Barchetta, F. Angelico, M. D. Ben et al., "Strong association between non alcoholic fatty liver disease (NAFLD) and low $25(\mathrm{OH})$ vitamin D levels in an adult population with normal serum liver enzymes," BMC Medicine, vol. 9, article 85, 2011.

[33] S. Vanlint, "Vitamin D and obesity," Nutrients, vol. 5, no. 3, pp. 949-956, 2013.

[34] I. González-Molero, G. Rojo-Martínez, S. Morcillo et al., "Hypovitaminosis D and incidence of obesity: a prospective study," European Journal of Clinical Nutrition, vol. 67, no. 6, pp. 680-682, 2013.

[35] A. Cordeiro, S. E. Pereira, C. J. Saboya, and A. Ramalho, "Association between 25(OH)D concentrations and metabolic syndrome components in class III obese subjects," International Journal of Medicine and Medical Sciences, vol. 48, no. 1, pp. 1597$1603,2015$.

[36] T. Tzotzas, F. G. Papadopoulou, K. Tziomalos et al., "Rising serum 25-hydroxy-vitamin D levels after weight loss in obese women correlate with improvement in insulin resistance," Journal of Clinical Endocrinology and Metabolism, vol. 95, no. 9, pp. 4251-4257, 2010.

[37] R. Jorde, M. Sneve, N. Emaus, Y. Figenschau, and G. Grimnes, "Cross-sectional and longitudinal relation between serum 25hydroxyvitamin D and body mass index: the Tromsø study," European Journal of Nutrition, vol. 49, no. 7, pp. 401-407, 2010.

[38] K. Rajakumar, J. De Las Heras, T. C. Chen, S. Lee, M. F. Holick, and S. A. Arslanian, "Vitamin D status, adiposity, and lipids in black American and Caucasian children," Journal of Clinical Endocrinology and Metabolism, vol. 96, no. 5, pp. 1560-1567, 2011.

[39] K. S. Vimaleswaran, D. J. Berry, C. Lu et al., "Causal relationship between obesity and vitamin D status: bi-directional Mendelian randomization analysis of multiple cohorts," PLoS Medicine, vol. 10, no. 2, Article ID e1001383, 2013.

[40] S. P. Bhatt, P. Nigam, A. Misra, R. Guleria, and M. A. Qadar Pasha, "Independent associations of low 25 hydroxy vitamin $\mathrm{D}$ and high parathyroid hormonal levels with nonalcoholic fatty liver disease in Asian Indians residing in north India," Atherosclerosis, vol. 230, no. 1, pp. 157-163, 2013.

[41] P. Trayhurn, "Hypoxia and adipose tissue function and dysfunction in obesity," Physiological Reviews, vol. 93, no. 1, pp. 1-21, 2013. 
[42] M. Vlasova, A. K. Purhonen, M. R. Jarvelin, E. Rodilla, J. Pascual, and K. H. Herzig, "Role of adipokines in obesityassociated hypertension," Acta Physiologica, vol. 200, no. 2, pp. $107-127,2010$

[43] S. Galic, J. S. Oakhill, and G. R. Steinberg, "Adipose tissue as an endocrine organ," Molecular and Cellular Endocrinology, vol. 316, no. 2, pp. 129-139, 2010.

[44] S. Cheng, J. M. Massaro, C. S. Fox et al., "Adiposity, cardiometabolic risk, and vitamin D status: the framingham heart study," Diabetes, vol. 59, no. 1, pp. 242-248, 2010.

[45] X. Sun, K. L. Morris, and M. B. Zemel, "Role of calcitriol and cortisol on human adipocyte proliferation and oxidative and inflammatory stress: a microarray study," Journal of Nutrigenetics and Nutrigenomics, vol. 1, no. 1-2, pp. 30-48, 2007.

[46] C. Ding, J. P. Wilding, and C. Bing, "1,25-dihydroxy vitamin D3 protects against macrophage-induced activation of NF kappa $\mathrm{B}$ and MAPK signalling and chemokine release in human adipocytes," PLOS ONE, vol. 8, no. 4, article e61707, 2013.

[47] L. Wamberg, K. B. Cullberg, L. Rejnmark, B. Richelsen, and S. B. Pedersen, "Investigations of the anti-inflammatory effects of vitamin $\mathrm{D}$ in adipose tissue: results from an in vitro study and a randomized controlled trial," Hormone and Metabolic Research, vol. 45, no. 6, pp. 456-462, 2013.

[48] S. C. Benoit, T. D. Hunter, D. M. Francis, and N. De La Cruz-Munoz, "Use of bariatric outcomes longitudinal database (BOLD) to study variability in patient success after bariatric surgery," Obesity Surgery, vol. 24, no. 6, pp. 936-943, 2014.

[49] M. A. Cabral, C. N. Borges, J. M. C. Maia, C. A. M. Aires, and F. Bandeira, "Prevalence of vitamin D deficiency during the summer and its relationship with sun exposure and skin phototype in elderly men living in the tropics," Clinical Interventions in Aging, vol. 8, pp. 1347-1351, 2013.

[50] N. M. van Schoor and P. Lips, "Worldwide vitamin D status," Best Practice and Research: Clinical Endocrinology and Metabolism, vol. 25, no. 4, pp. 671-680, 2011.

[51] D. A. Wahl, C. Cooper, P. R. Ebeling et al., "A global representation of vitamin D status in healthy populations," Archives of Osteoporosis, vol. 7, no. 1-2, pp. 155-172, 2012.

[52] C. Palacios and L. Gonzalez, "Is vitamin D deficiency a major global public health problem?" Journal of Steroid Biochemistry and Molecular Biology, vol. 144, no. Part A, pp. 138-145, 2014.

[53] V. M. Lopes, J. R. Lopes, J. P. Brasileiro et al., "Highly prevalence of vitamin $\mathrm{D}$ deficiency among Brazilian women of reproductive age," Archives of Endocrinology and Metabolism, vol. 61, no. 1, pp. 21-27, 2017.

[54] P. D. Clare Grace, R. Vincent, and S. J. Aylwin, "High prevalence of vitamin D insufficiency in a United Kingdom urban morbidly obese population: implications for testing and treatment," Surgery for Obesity and Related Diseases, vol. 10, no. 2, pp. 355360, 2014.

[55] H. D. Vo, J. Xu, S. S. Rabinowitz, S. E. Fisher, and S. M. Schwarz, "The liver in pediatric gastrointestinal disease," Journal of Pediatric Gastroenterology and Nutrition, vol. 59, no. 3, pp. 288299, 2014.

[56] P. Paschos and K. Paletas, "Non alcoholic fatty liver disease and metabolic syndrome," Hippokratia, vol. 13, no. 1, pp. 9-19, 2009.

[57] G. V. Chaves, D. S. de Souza, S. E. Pereira, C. J. Saboya, and W. A. Ferreira Peres, "Association between non-alcoholic fatty liver disease and liver function/injury markers with metabolic syndrome components in class III obese individuals," Revista da Associação Médica Brasileira, vol. 58, no. 3, pp. 288-293, 2012.
[58] G. Bedogni, L. Miglioli, F. Masutti, C. Tiribelli, G. Marchesini, and S. Bellentani, "Prevalence of and risk factors for nonalcoholic fatty liver disease: the dionysos nutrition and liver study," Hepatology, vol. 42, no. 1, pp. 44-52, 2005.

[59] M. Bayard, J. Holt, and E. Boroughs, "Nonalcoholic fatty liver disease," American Family Physician, vol. 73, no. 11, pp. 19611968,2006 


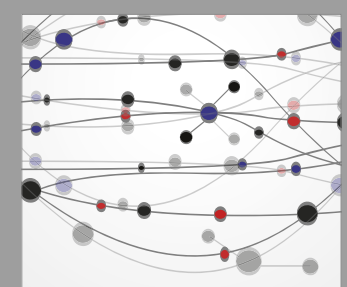

The Scientific World Journal
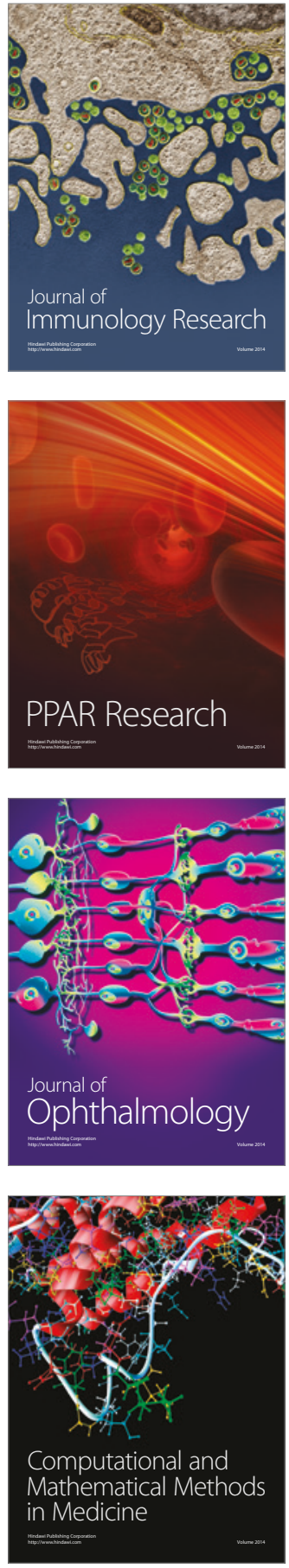

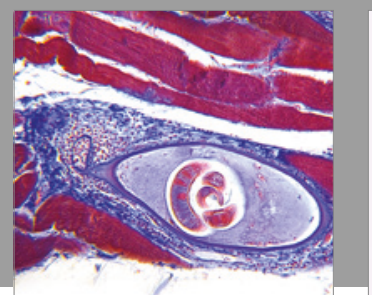

Gastroenterology Research and Practice
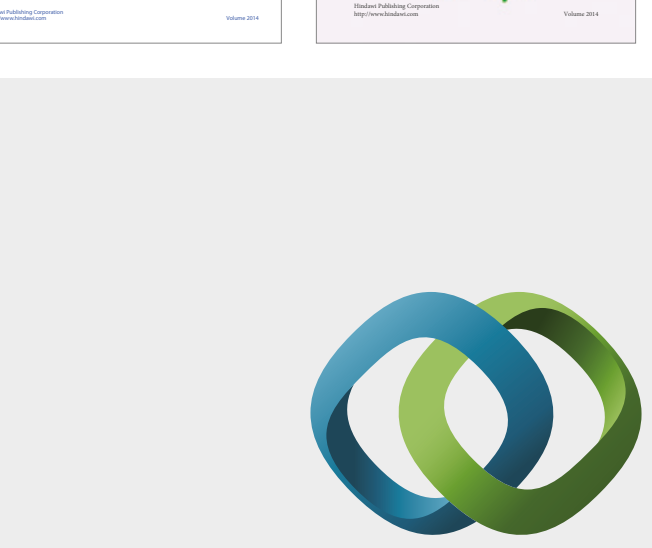

\section{Hindawi}

Submit your manuscripts at

https://www.hindawi.com
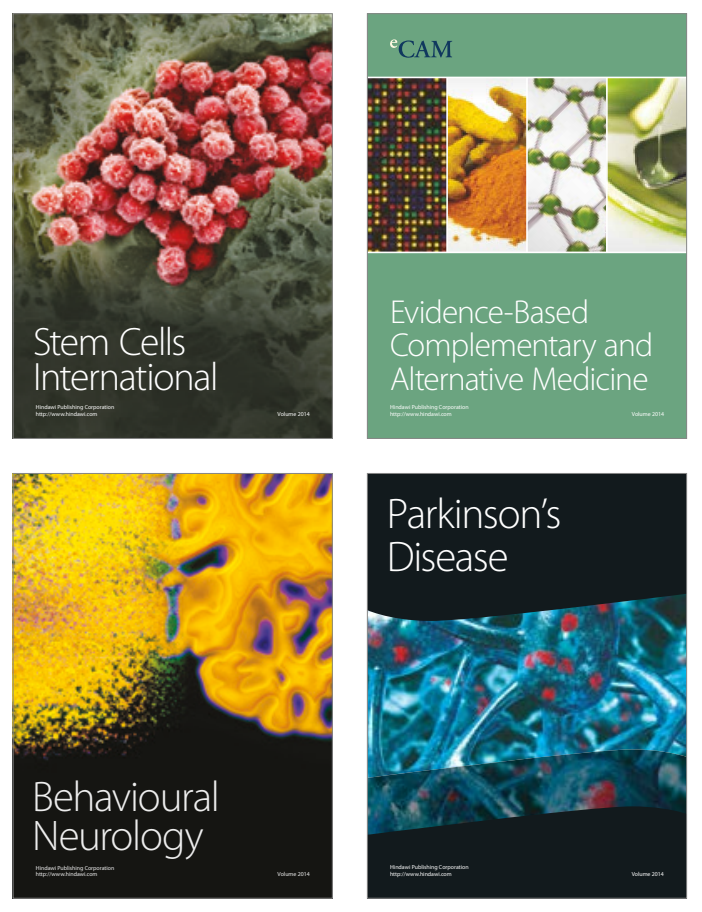
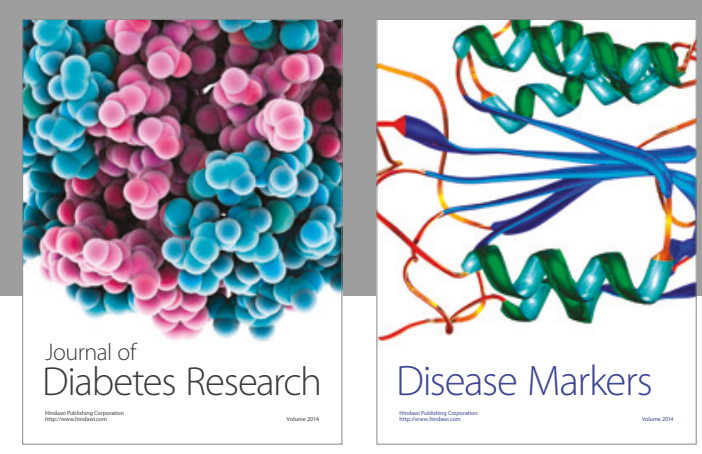

Disease Markers
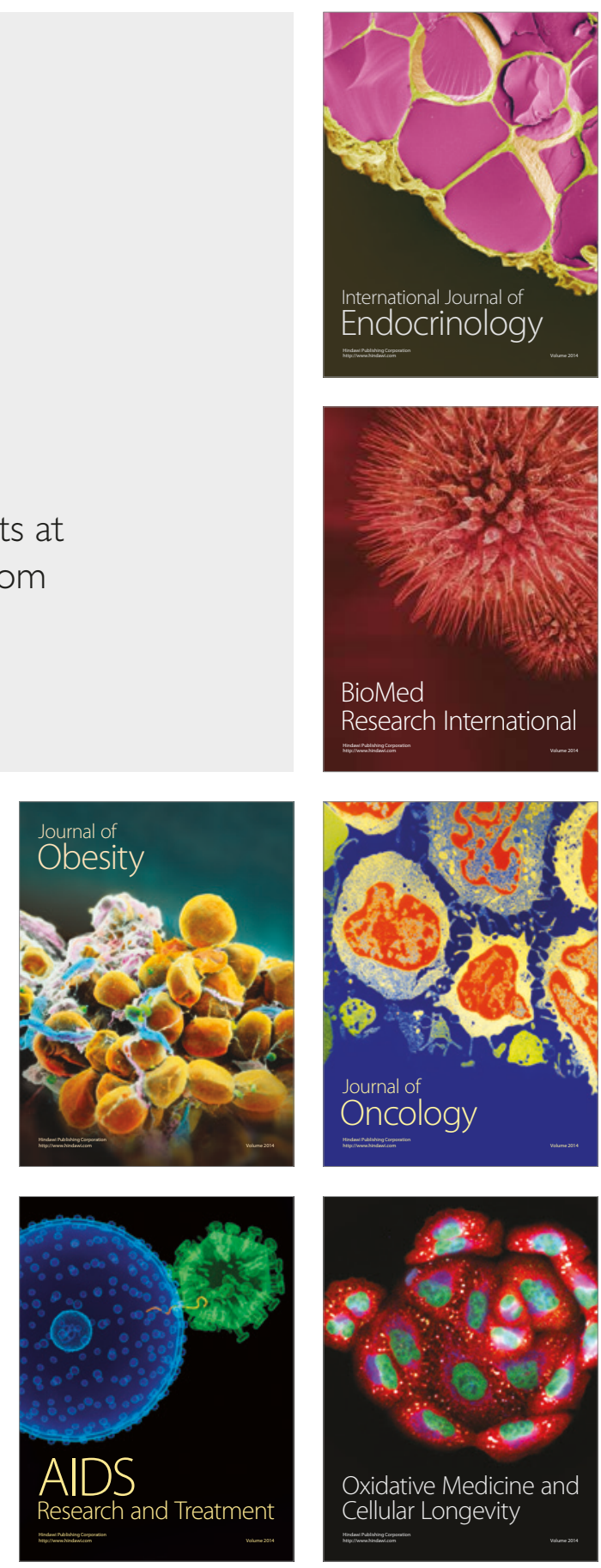